VOL. $2(1970), 369-399$.

\title{
Averaging and integral manifolds (II)
}

\section{K. J. Palmer}

In the first part of this paper (written jointly with W.A. Coppel) the existence and properties of an integral manifold were established for the system

$$
\begin{aligned}
& x^{\prime}=f(t, x, y) \\
& y^{\prime}=A(t) y+g(t, x, y)
\end{aligned}
$$

where $f$ and $g$ are "integrally small". In this second part of the paper the stability properties of the integral manifold are investigated. Solutions are found which are bounded on the positive half of the real line and it is shown that these solutions approach the manifold exponentially and, moreover, that they are asymptotic to particular solutions on the manifold.

1.

This paper is a continuation of Coppel and Palmer [1]. We consider once more the system of differential equations

$$
x^{\prime}=f(t, x, y)
$$

$$
y^{\prime}=A(t) y+g(t, x, y),
$$

where $x \in R^{m}, y \in R^{n}, '=d / d t$, and where the linear equation

$$
y^{\prime}=A(t) y
$$

has a fundamental matrix $Y(t)$ such that

Received 23 February 1970. Communicated by W.A. Coppel. The author would like to thank his supervisor Mr Andrew Coppel for his generous help in the preparation of this paper and for many suggestions for improvement. The author was supported by a CSIRO Postgraduate Studentship. 


$$
\begin{aligned}
\left|Y(t) P Y^{-1}(s)\right| \leq K e^{-2 \alpha(t-s)} & \text { for } t \geq s, \\
\left|Y(t)(I-P) Y^{-1}(s)\right| \leq K e^{-2 \alpha(s-t)} & \text { for } s \geq t,
\end{aligned}
$$

where the matrix $P$ is a projection and $K, \alpha$ are positive constants. However, instead of supposing this system to be defined on the whole real line we now suppose it to be defined on a half-line $[\tau, \infty)$. This enables us to impose a 'partial' initial condition on $y$. Given a vector $\xi \in R^{m}$ and a vector $\eta \in R^{n}$ we look for a solution $x(t)=x(t, \xi, \eta, \tau)$, $y(t)=y(t, \xi, \eta, \tau)$ of (1) such that $y(t)$ is bounded and

$$
x(\tau)=\xi, P(\tau) y(\tau)=P(\tau) \eta,
$$

where $P(\tau)$ denotes the projection $Y(\tau) P Y^{-1}(\tau)$. For each fixed $\tau, \xi$ the set of all points $y(\tau, \xi, \eta, \tau)$ defines a submanifold of $R^{n}$, of dimension equal to the rank of $P$, such that only solutions starting from this submanifold remain bounded. The argument of [1] carries over to the present problem with some complication but without essential change, so much of the detail will be omitted. However the treatment of [1] is improved in that no use is made of the roughness property of exponential dichotomies (Lemma 2 of [1]). This means that everything carries over to the case where $R^{m}$ and $R^{n}$ are replaced by arbitrary Banach spaces. In $\$ 3$ the present results are used to discuss the stability of the integral manifold considered in the previous paper. The concluding section contains a discussion of related work by other authors and some remarks on the smoothness of the integral manifold.

We again set

$$
\|f\|=\sup _{t \geq \tau}\left\{e^{-\beta(t-\tau)}|f(t)|\right\} .
$$

The following two lemmas correspond to Lemmas 4 and 5 of [1] and are proved in a similar way.

LEMMA 1. Let $A(t)$ be a continuous matrix function such that the linear equation (2) has a fundomental matrix $y(t)$ satisfying (3). If $f(t)$ is a continuous vector function such that $\|f\|<\infty$, where $|\beta|<2 \alpha$, then the inhomogeneous equation 


$$
y^{\prime}=A(t) y+f(t)
$$

has a unique solution $y(t)$ such that $P(\tau) y(\tau)=0$ and $\|y\|<\infty$. Moreover

$$
\|y\| \leq\left\{(2 \alpha+\beta)^{-1}+(2 \alpha-\beta)^{-1}\right\} K\|f\|
$$

Thus if $|\beta| \leq \alpha$ then

$$
\|y\| \leq \frac{4}{3} \alpha^{-1} K\|f\|
$$

The solution $y(t)$ is given explicitly by

$$
y(t)=\int_{\tau}^{t} Y(t) P Y^{-1}(s) f(s) d s-\int_{t}^{\infty} Y(t)(I-P) Y^{-1}(s) f(s) d s .
$$

If we add $Y(t) P Y^{-1}(\tau) \eta$ to the right side we obtain the unique solution $y(t)$ of $(4)$ with $P(\tau) y(\tau)=P(\tau) \eta$ and $\|y\|<\infty$.

LEMMA 2. Let $A(t)$ be a continuous matrix function such that $|A(t)| \leq N$ for $t \geq \tau$, where $N \geq 1$, and suppose the linear equation (2) has a fundamental matrix $Y(t)$ satisfying (3). If $f(t)$ is a continuous vector function such that

$$
\left|\int_{t}^{t+h} f(s) d s\right| \leq r e^{B(t-\tau)}
$$

for $0 \leq h \leq 1$ and $t \geq \tau$, where $|\beta|<2 \alpha$, then the inhomogeneous equation (4) has a unique solution $y(t)$ such that $P(\tau) y(\tau)=0$ and $\|y\|<\infty$. Moreover

$$
\|y\| \leq\left\{\frac{1}{1-e^{-(2 \alpha+\beta)}}+\frac{1}{1-e^{-(2 \alpha-\beta)}}+\frac{N}{2 \alpha+\beta}+\frac{N}{2 \alpha-\beta}\right\} K r .
$$

Thus if $|\beta| \leq \alpha$ then

$$
\begin{aligned}
\|y\| & \leq\left(2 \gamma+\frac{4}{3} \alpha^{-1} N\right) K r \\
& \leq 4 N K \gamma r,
\end{aligned}
$$

where $\gamma=\left(1-e^{-\alpha}\right)^{-1}$.

We impose the same conditions on the system (1) as in the previous 
paper, except that the functions involved need only be defined for $t \geq \tau$. That is, we assume $f$ and $g$ are continuous vector functions with continuous partial derivatives in $x$ and $y$ such that

$$
\begin{gathered}
|f(t, x, y)| \leq N,|g(t, x, y)| \leq N, \\
\left|f_{x}(t, x, y)\right| \leq N, \\
\left|f_{x}\left(t, x_{1}, y_{1}\right)-f_{x}\left(t, x_{2}, y_{2}\right)\right| \leq L\left[\left|x_{1}-x_{2}\right|+\left|y_{1}-y_{2}\right|\right],
\end{gathered}
$$

where $N \geq 1$, and the same inequalities with $f_{x}$ replaced by $f_{y}, g_{x}$, $g_{y} \cdot \quad$ Furthermore we assume

$$
\left|\int_{t_{1}}^{t_{2}} g(t, x, 0) d t\right| \leq q \text { for all } x \text { if }\left|t_{2}-t_{1}\right| \leq 1 \text {, }
$$

and the same inequality with $g$ replaced by $f_{x}, g_{x}, g_{y}$. Finally we suppose that $A(t)$ is a continuous matrix function, with $|A(t)| \leq N$, such that the linear equation (2) has a fundamental matrix $Y(t)$ satisfying ( 3 ). Under these assumptions we will prove

THEOREM 1. For any $\beta\left(0<\beta \leq \frac{1}{2} \alpha\right)$ there exists a positive constant $\mu_{0}=\mu_{0}(N, K, L, \alpha, \beta)$ such that if $\mu \leq \mu_{0}$ and if $q \leq q_{0}(N, K, L, \alpha, \beta, \mu)$ then for any vector $n$ with $|\eta|<\mu / 4 K$ the system of differential equations

$$
\begin{aligned}
& x^{\prime}=f(t, x, y) \\
& y^{\prime}=A(t) y+g(t, x, y)
\end{aligned}
$$

has a unique solution $x(t)=x(t, \xi, \eta, \tau), y(t)=y(t, \xi, \eta, \tau)$ for which

$$
x(\tau)=\xi, \quad P(\tau) y(\tau)=P(\tau) n, \quad|y(t)| \leq \mu \text { for } t \geq \tau .
$$

Moreover the partial derivatives $x_{\xi}, x_{n}, y_{\xi}, y_{n}$ exist and satisfy 


$$
\begin{aligned}
& \left|x_{\xi}(t, \xi, \eta, \tau)\right| \leq 2 e^{\beta(t-\tau)}, \quad\left|x_{\eta}(t, \xi, \eta, \tau)\right| \leq 2 C K e^{\beta(t-\tau)}, \\
& \left|y_{\xi}(t, \xi, n, \tau)\right| \leq 2 C^{-1} e^{\beta(t-\tau)},\left|y_{n}(t, \xi, n, \tau)\right| \leq 2 K e^{\beta(t-\tau)}, \\
& \left|x_{\xi}\left(t, \xi_{1}, n_{1}, \tau\right)-x_{\xi}\left(t, \xi_{2}, n_{2}, \tau\right)\right| \leq C D\left[\left|\xi_{1}-\xi_{2}\right|+C K\left|n_{1}-n_{2}\right|\right] e^{2 \beta(t-\tau)} \text {, } \\
& \left|y_{\xi}\left(t, \xi_{1}, n_{1}, \tau\right)-y_{\xi}\left(t, \xi_{2}, n_{2}, \tau\right)\right| \leq D\left[\left|\xi_{1}-\xi_{2}\right|+C K\left|n_{1}-n_{2}\right|\right] e^{2 \beta(t-\tau)}, \\
& \left|x_{n}\left(t, \xi_{1}, n_{1}, \tau\right)-x_{n}\left(t, \xi_{2}, \eta_{2}, \tau\right)\right| \leq c^{2} D K\left[\left|\xi_{1}-\xi_{2}\right|+C K\left|n_{1}-n_{2}\right|\right] e^{2 \beta(t-\tau)} \text {, } \\
& \left|y_{n}\left(t, \xi_{1}, \eta_{1}, \tau\right)-y_{n}\left(t, \xi_{2}, \eta_{2}, \tau\right)\right| \leq C D K\left[\left|\xi_{1}-\xi_{2}\right|+C K\left|\eta_{1}-\eta_{2}\right|\right] e^{2 B(t-\tau)} \text {, }
\end{aligned}
$$

where $C=4 N\left(1-e^{-\beta}\right)^{-1}$ and $D=8 L\left(N^{-1}+2 \alpha^{-1} K\right)$.

Set

$$
\nu=(8 N)^{-1}, \quad R=4 N K \alpha^{-1} e^{\alpha}, \quad \gamma=\left(1-e^{-\alpha}\right)^{-1} \text {, }
$$

and choose $\mu_{0}>0$ so that

$$
64 \mu_{0} \operatorname{LNC}(R+\gamma) \leq 1 \text {. }
$$

Next for any $\mu \quad\left(0<\mu \leq \mu_{0}\right)$ we choose $p_{0}>0$ so that

$$
64 p_{0} N C(R+\nu) \leq 1, \quad 24 p_{0}(1+\mu) \gamma N^{2} K \leq \mu .
$$

Then we take

$$
q_{0}=\frac{1}{2} p_{0}^{2} \min \left[\left(p_{0}+L N\right)^{-1},\left(p_{0}+N^{2}\right)^{-1}\right]
$$

Let $x(t)$ and $y(t)$ be continuously differentiable functions such that

$$
\left|x^{\prime}(t)\right| \leq N,|y(t)| \leq \mu, \quad\left|y^{\prime}(t)\right| \leq(1+\mu) N \text { for } t \geq \tau .
$$

Put

$$
\hat{x}(t)=\xi+\int_{\tau}^{t} f[s, x(s), y(s)] d s
$$

and let $\hat{y}(t)$ denote the bounded solution of the equation

$$
\hat{y}^{\prime}=A(t) \hat{y}+g[t, x(t), y(t)]
$$

such that $P(\tau) \hat{y}(\tau)=P(\tau) \eta$. Such a solution exists and is unique, by 
Lemma 1 with $\beta=0$. If we write

$$
\begin{aligned}
& f(t, x, y)=f(t, x, 0)+f_{y}(t, x, 0) y+F(t, x, y) \\
& g(t, x, y)=g(t, x, 0)+g_{y}(t, x, 0) y+G(t, x, y)
\end{aligned}
$$

then

$$
|F(t, x, y)| \leq \frac{1}{2} L|y|^{2}, \quad|G(t, x, y)| \leq \frac{1}{2} L|y|^{2},
$$

and if $\left|y_{1}\right| \leq \mu,\left|y_{2}\right| \leq \mu$

$$
\begin{aligned}
& \left|F\left(t, x_{1}, y_{1}\right)-F\left(t, x_{2}, y_{2}\right)\right| \leq 2 \mu L\left[\left|x_{1}-x_{2}\right|+\left|y_{1}-y_{2}\right|\right], \\
& \left|G\left(t, x_{1}, y_{1}\right)-G\left(t, x_{2}, y_{2}\right)\right| \leq 2 \mu L\left[\left|x_{1}-x_{2}\right|+\left|y_{1}-y_{2}\right|\right] .
\end{aligned}
$$

Thus

$$
|G[t, x(t), y(t)]| \leq \frac{1}{2} \mu^{2} L
$$

For $\left|t_{2}-t_{1}\right| \leq 1$ we have, by Lemma 3 of [1],

$$
\left|\int_{t_{1}}^{t_{2}} g[t, x(t), 0] d t\right| \leq p_{0}
$$

and, by Lemma 7 of [1] with $\beta=0$,

$$
\left|\int_{t_{1}}^{t_{2}} g_{y}[t, x(t), 0] y(t) d t\right| \leq p_{0}\{\mu+(1+\mu) N\} \text {. }
$$

Therefore, since $N \geq 1$,

$$
\left|\int_{t_{1}}^{t_{2}}\left\{g[t, x(t), 0]+g_{y}[t, x(t), 0] y(t)\right\} d t\right| \leq(1+\mu) 2 N p_{0} .
$$

It follows from the present Lemmas 1 and 2 , with $\beta=0$, and from the superposition principle that

$$
\begin{aligned}
|\hat{y}(t)| & \leq\left|Y(t) P Y^{-1}(\tau) n\right|+\frac{1}{2} \mu^{2} L \cdot \alpha^{-1} K+(1+\mu) 2 N p_{0} \cdot\left(2 \gamma+N \alpha^{-1}\right) K \\
& \leq K|n|+\frac{1}{2} \mu^{2} \gamma K L+6(1+\mu) \gamma N^{2} K p_{0} \\
& \leq \frac{1}{4} \mu+\frac{1}{4} \mu+\frac{1}{4} \mu=\frac{3}{4} \mu .
\end{aligned}
$$

Hence $\left|\hat{y}^{\prime}(t)\right| \leq(1+\mu) N$, and it is obvious that $\left|\hat{x}^{\prime}(t)\right| \leq N$. 
For a fixed $\beta$ such that $0<\beta \leq \alpha$ let $B$ denote the set of all pairs $(x, y)$, where $x=x(t)$ and $y=y(t)$ are continuously differentiable functions for $t \geq \tau$ with $\|x\|+\left\|x^{\prime}\right\|+\|y\|+\left\|y^{\prime}\right\|<\infty$. The set $B$ becomes a Banach space if we define

$$
\begin{aligned}
\left(x_{1}+x_{2}, y_{1}+y_{2}\right) & =\left(x_{1}, y_{1}\right)+\left(x_{2}, y_{2}\right) \\
\lambda(x, y) & =(\lambda x, \lambda y) \\
|(x, y)| & =\|x\|+v\left\|x^{\prime}\right\|+c\|y\|+v\left\|y^{\prime}\right\|,
\end{aligned}
$$

where $v, C$ are the positive constants defined above. The set $S$ of all pairs $(x, y)$ in $B$ satisfying $(6)$ is a closed subset of $B$ and, by what we have just proved, the transformation $T:(x, y) \rightarrow(\hat{x}, \hat{y})$ maps $S$ into itself. We show next that $T$ is a contraction on $S$.

Let $\left(x_{1}, y_{1}\right)$ and $\left(x_{2}, y_{2}\right)$ be any two points in $S$, and let $\left(\hat{x}_{1}, \hat{y}_{1}\right)$ and $\left(\hat{x}_{2}, \hat{y}_{2}\right)$ be their images under $T$. We set

$$
(z, w)=\left(x_{1}, y_{1}\right)-\left(x_{2}, y_{2}\right),(\hat{z}, \hat{w})=\left(\hat{x}_{1}, \hat{y}_{1}\right)-\left(\hat{x}_{2}, \hat{y}_{2}\right) .
$$

Then

$$
\hat{\boldsymbol{z}}(t)=\int_{\tau}^{t}\left\{f\left[s, x_{1}(s), y_{1}(s)\right]-f\left[s, x_{2}(s), y_{2}(s)\right]\right\} d s .
$$

By exactly the same argument as in the previous paper it follows that

$$
\begin{aligned}
\|\hat{z}\| & \leq H\left\{3 \mu L[\|z\|+\|w\|]+N\|w\|+p_{0}\left[\|z\|+\left\|z^{\prime}\right\|\right]\right\}, \\
\left\|\hat{z}^{\prime}\right\| & \leq N[\|z\|+\|w\|],
\end{aligned}
$$

where $H=\left(1-e^{-\beta}\right)^{-1}$. The difference $\hat{w}(t)=\hat{y}_{1}(t)-\hat{y}_{2}(t)$ is a bounded solution, with $P(\tau) \hat{w}(\tau)=0$, of the equation

$$
\hat{w}^{\prime}=A(t) \hat{\omega}+\varphi(t)+\psi(t),
$$

where

$$
\begin{aligned}
\varphi(t)= & g\left[t, x_{1}(t), 0\right]-g\left[t, x_{2}(t), 0\right]+g_{y}\left[t, x_{1}(t), 0\right] w(t), \\
\psi(t)=G\left[t, x_{1}(t), y_{1}(t)\right]-G\left[t, x_{2}(t), y_{2}(t)\right] & \\
& +\left\{g_{y}\left[t, x_{1}(t), 0\right]-g_{y}\left[t, x_{2}(t), 0\right]\right\} y_{2}(t) .
\end{aligned}
$$

It follows at once that

$$
|\psi(t)| \leq 3 \mu L[|z(t)|+|\omega(t)|] .
$$


Also, using Lemma 7 of [1] we obtain for $0 \leq h \leq 1$

$$
\left|\int_{t}^{t+h} \varphi(s) d s\right| \leq p_{0} \beta^{-1}\left(e^{\beta}-1\right)\left[\|z\|+\left\|z^{\prime}\right\|+\|w\|+\left\|w^{\prime}\right\|\right] e^{\beta(t-\tau)} .
$$

By Lemmas 1 and 2 and the superposition principle it follows that

$$
\begin{aligned}
\|\hat{w}\| & \leq \frac{4}{3} \alpha^{-1} K \cdot 3 \mu L[\|z\|+\|w\|]+4 N K \gamma p_{0} \beta^{-1}\left(e^{\beta}-1\right)\left[\|z\|+\left\|z^{\prime}\right\|+\|w\|+\left\|w^{\prime}\right\|\right] \\
& \leq 4 \alpha^{-1} \mu L K[\|z\|+\|w\|]+p_{0} R\left[\|z\|+\left\|z^{\prime}\right\|+\|w\|+\left\|w^{\prime}\right\|\right] .
\end{aligned}
$$

Also

$$
\left\|\hat{w}^{\prime}\right\| \leq N\|\hat{w}\|+N[\|z\|+\|w\|]
$$

Combining these estimates and using the inequalities imposed on $\mu$ and $p_{0}$ we get

$$
|(\hat{z}, \hat{w})| \leq \frac{1}{2}|(z, w)|
$$

Thus the mapping $T$ is a contraction. Its fixed point is the required solution $x(t, \xi, \eta, \tau), y(t, \xi, \eta, \tau)$.

If $\left(x_{1}, y_{1}\right)$ and $\left(x_{2}, y_{2}\right)$ are the fixed points corresponding to the initial values $\left(\xi_{1}, \eta_{1}\right)$ and $\left(\xi_{2}, n_{2}\right)$ respectively we obtain in the same way

$$
|(z, w)| \leq\left|\xi_{1}-\xi_{2}\right|+C K\left|n_{1}-n_{2}\right|+\frac{1}{2}|(z, w)|
$$

and hence

$$
|(z, w)| \leq 2\left|\xi_{1}-\xi_{2}\right|+2 C K\left|n_{1}-n_{2}\right|
$$

Thus

$$
\begin{aligned}
& \left|x\left(t, \xi_{1}, \eta_{1}, \tau\right)-x\left(t, \xi_{2}, n_{2}, \tau\right)\right| \leq 2\left[\left|\xi_{1}-\xi_{2}\right|+c k\left|\eta_{1}-\eta_{2}\right|\right] e^{\beta(t-\tau)}, \\
& \left|y\left(t, \xi_{1}, \eta_{1}, \tau\right)-y\left(t, \xi_{2}, n_{2}, \tau\right)\right| \leq 2\left[c^{-l .1}\left|\xi_{1}-\xi_{2}\right|+k\left|\eta_{1}-\eta_{2}\right|\right] e^{\beta(t-\tau)} .
\end{aligned}
$$

Similarly let $\left(x_{1}, y_{1}\right)$ and $\left(x_{2}, y_{2}\right)$ be the fixed points corresponding to the initial values $\xi, n$ at times $\tau$ and $\tau+h$ respectively, where $h>0$. If we again set $(z, w)=\left(x_{1}, y_{1}\right)-\left(x_{2}, y_{2}\right)$ then 
Averaging and integral manifolds

377

$$
\begin{aligned}
z(t)=\int_{\tau}^{\tau+h} f\left[s, x_{2}(s), y_{2}(s)\right] d s & \\
& +\int_{\tau}^{t}\left\{f\left[s, x_{1}(s), y_{1}(s)\right]-f\left[s, x_{2}(s), y_{2}(s)\right]\right\} d s
\end{aligned}
$$

and

$$
\begin{aligned}
w(t)=Y(t) P\left[Y^{-1}(\tau)-Y^{-1}(\tau+h)\right] \eta+\int_{\tau}^{\tau+h} Y(t) P Y^{-1}(s) g\left[s, x_{2}(s), y_{2}(s)\right] d s \\
+\int_{\tau}^{t} Y(t) P Y^{-1}(s) \zeta(s) d s-\int_{t}^{\infty} Y(t)(I-P) Y^{-1}(s) \zeta(s) d s,
\end{aligned}
$$

where

$$
\zeta(t)=g\left[t, x_{1}(t), y_{1}(t)\right]-g\left[t, x_{2}(t), y_{2}(t)\right] .
$$

We have

$$
\begin{aligned}
\left|\int_{\tau}^{\tau+h} f\left[s, x_{2}(s), y_{2}(s)\right] d s\right| & \leq N h, \\
\left|Y(t) P\left[Y^{-1}(\tau)-Y^{-1}(\tau+h)\right]\right| & =\left|\int_{\tau}^{\tau+h} Y(t) P Y^{-1}(s) A(s) d s\right| \\
& \leq \int_{\tau}^{\tau+h} K e^{N(s-\tau)} N d s \\
& \leq K N e^{N h},
\end{aligned}
$$

and similarly

$$
\left|\int_{\tau}^{\tau+h} Y(t) P Y^{-1}(s) g\left[s, x_{2}(s), y_{2}(s)\right] d s\right| \leq K N e^{N h} h .
$$

It follows that

(9)

$$
|(z, w)| \leq 2 N h+4 C K N e^{N h} h
$$

Thus

$$
\begin{aligned}
& |x(t, \xi, \eta, \tau+h)-x(t, \xi, \eta, \tau)| \leq 2 N\left(1+2 C K e^{N h}\right) h e^{\beta(t-\tau)} \\
& |y(t, \xi, \eta, \tau+h)-y(t, \xi, \eta, \tau)| \leq \frac{1}{2}\left(1-e^{-\beta}\right)\left(1+2 C K e^{N h}\right) h e^{\beta(t-\tau)} .
\end{aligned}
$$

https://doi.org/10.1017/S0004972700042064 Published online by Cambridge University Press 
This shows, a posteriori, that if $0 \leq h \leq h_{0}$, the solutions $x(t, \xi, \eta, \tau+h), y(t, \xi, \eta, \tau+h)$ can be continued over the interval $[\tau, \tau+h]$ without leaving the region $|y|<\mu$.

2.

To prove the existence and Lipschitzian nature of the partial derivatives we consider first a linear system of differential equations

$$
\begin{aligned}
& x^{\prime}=F_{1}(t) x+E_{2}(t) y+\chi(t) \\
& y^{\prime}=G_{1}(t) x+\left[A(t)+G_{2}(t)\right] y+\zeta(t),
\end{aligned}
$$

on $[\tau, \infty)$, where the matrix functions $F_{k}, G_{k}(k=1,2)$ are continuous and bounded by $N$, and the vector functions $\chi, \zeta$ are continuous with $\|x\|<\infty,\|\zeta\|<\infty$. We assume also that for $\left|t_{2}-t_{1}\right| \leq 1$

$$
\left|\int_{t_{1}}^{t_{2}} F_{1}(t) d t\right|,\left|\int_{t_{1}}^{t_{2}} G_{1}(t) d t\right|,\left|\int_{t_{1}}^{t_{2}} G_{2}(t) d t\right| \leq r .
$$

We wish to show that if $r$ is so small that

$$
32 N C(R+v) r \leq 1 \text {, }
$$

then the system (10) has a unique solution $x(t), y(t)$ in $B$ such that $x(\tau)=\xi$ and $P(\tau) y(\tau)=P(\tau) \eta$.

For any $(x, y)$ in $B$ set

$$
\hat{x}(t)=\xi+\int_{\tau}^{t}\left\{F_{1}(s) x(s)+F_{2}(s) y(s)+\chi(s)\right\} d s
$$

and let $\hat{y}(t)$ denote the unique solution with $P(\tau) \hat{y}(\tau)=P(\tau) \eta$ and $\|\hat{y}\|<\infty$ of the equation

$$
\hat{y}^{\prime}=A(t) \hat{y}+G_{1}(t) x(t)+G_{2}(t) y(t)+\zeta(t) .
$$

Using Lemma 2 and proceeding as in [1] we obtain, with the same notation as above, 


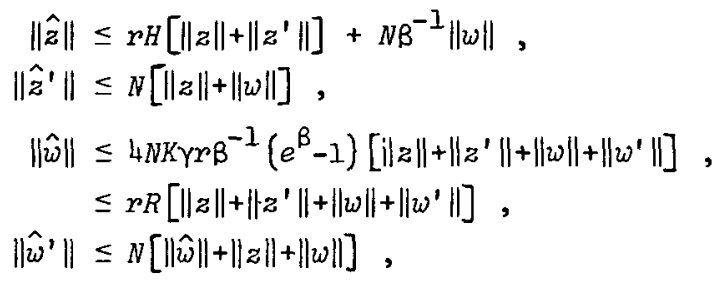

and hence

$$
|(\hat{z}, \hat{w})| \leq \frac{1}{2}|(z, w)|
$$

Therefore the mapping $(x, y) \rightarrow(\hat{x}, \hat{y})$ is a contraction, and the result follows. Moreover the solution $x(t), y(t)$ satisfies the inequality

$$
|(x, y)| \leq 2|\xi|+2 C K|n|+N^{-1} C\|x\|+2\left(2 \alpha^{-1} C K+\nu\right)\|\zeta\|
$$

If

$$
\begin{aligned}
& F_{1}(t)=f_{x}[t, x(t, \xi, \eta, \tau), y(t, \xi, \eta, \tau)], \\
& F_{2}(t)=f_{y}[t, x(t, \xi, n, \tau), y(t, \xi, \eta, \tau)], \\
& G_{1}(t)=g_{x}[t, x(t, \xi, n, \tau), y(t, \xi, \eta, \tau)], \\
& G_{2}(t)=g_{y}[t, x(t, \xi, \eta, \tau), y(t, \xi, \eta, \tau)],
\end{aligned}
$$

then we can take $r=p_{0}+\mu_{0} L$ and the inequality (12) is satisfied. Let $X_{1}(t), Y_{1}(t)$ denote the corresponding solution in $B$ of the matrix system

$$
\begin{aligned}
& X^{\prime}=F_{1}(t) X+F_{2}(t) Y \\
& Y^{\prime}=G_{1}(t) X+\left[A(t)+G_{2}(t)\right] Y
\end{aligned}
$$

with $X_{1}(\tau)=I, P(\tau) Y_{1}(\tau)=0$. Then by $(13)$,

$$
\left\|X_{1}\right\|+C\left\|Y_{1}\right\| \leq 2 \text {. }
$$

As in [1] we can show that the partial derivatives $x_{\xi}(t, \xi, n, \tau)$, $y_{\xi}(t, \xi, n, \tau)$ exist and equal $X_{1}(t), y_{1}(t)$ respectively. Similarly, if $X_{2}(t), Y_{2}(t)$ is the solution in $B$ of $(14)$ with $X_{2}(\tau)=0$, $P(\tau) Y_{2}(\tau)=P(\tau)$ then

$$
\left\|X_{2}\right\|+C\left\|Y_{2}\right\| \leq 2 C K
$$


and we can show that the partial derivatives $x_{\eta}(t, \xi ; \eta, \tau)$, $y_{n}(t, \xi, \eta, \tau)$ exist and equal $X_{2}(t), y_{2}(t)$ respectively.

It remains to show that the partial derivatives satisfy Lipschitz conditions. If we set

$$
\tilde{X}_{1}(t)=x_{\xi}(t, \tilde{\xi}, \tilde{n}, \tau), \tilde{Y}_{1}(t)=y_{\xi}(t, \tilde{\xi}, \tilde{n}, \tau)
$$

then $(Z, W)=\left(X_{1}, Y_{1}\right)-\left(\tilde{X}_{1}, \tilde{Y}_{1}\right)$ is the solution in $B$ of a system (10) with

$$
\begin{aligned}
& \chi(t)=\left[F_{1}(t)-\tilde{F}_{1}(t)\right] \tilde{X}_{1}(t)+\left[F_{2}(t)-\tilde{F}_{2}(t)\right] \tilde{Y}_{1}(t) \\
& \zeta(t)=\left[G_{1}(t)-\tilde{G}_{1}(t)\right] \tilde{X}_{1}(t)+\left[G_{2}(t)-\tilde{G}_{2}(t)\right] \tilde{Y}_{1}(t) .
\end{aligned}
$$

By (8) and (15)

$$
\begin{aligned}
|x(t)|,|\zeta(t)| & \leq L[|z(t)|+|w(t)|]\left[\left|\tilde{X}_{1}(t)\right|+\left|\tilde{Y}_{1}(t)\right|\right] \\
& \leq 4 L[|\xi-\tilde{\xi}|+c K|n-\tilde{\eta}|] e^{2 \beta(t-\tau)} .
\end{aligned}
$$

Put

$$
\|f\|_{2}=\sup _{t \geq \tau}\left\{e^{-2 \beta(t-\tau)}|f(t)|\right\} .
$$

Since $Z(\tau)=0$ and $P(\tau) W(\tau)=0$ it follows from (13) that

$$
\|z\|_{2}+C_{2}\|w\|_{2} \leq 2 C_{2}\left(N^{-1}+2 \alpha^{-1} K\right) \cdot 4 L[|\xi-\tilde{\xi}|+C K|n-\tilde{n}|]
$$

Therefore, since $C_{2}=4 N\left(1-e^{-2 B}\right)^{-1} \leq C$,

$$
\begin{aligned}
& \left|x_{\xi}(t, \xi, n, \tau)-x_{\xi}(t, \tilde{\xi}, \tilde{\eta}, \tau)\right| \leq C D[|\xi-\tilde{\xi}|+C K|\eta-\tilde{n}|] e^{2 \beta(t-\tau)} \\
& \left|y_{\xi}(t, \xi, n, \tau)-y_{\xi}(t, \tilde{\xi}, \tilde{\eta}, \tau)\right| \leq D[|\xi-\tilde{\xi}|+C K|\eta-\tilde{n}|] e^{2 \beta(t-\tau)} .
\end{aligned}
$$

The Lipschitz conditions for $x_{\eta}, y_{\eta}$ are proved similarly, and thus the proof of Theorem 1 is complete.

In the same way, for any $h\left(0 \leq h \leq h_{0}\right)$ the functions

$$
\begin{aligned}
& z(t)=x_{\xi}(t, \xi, \eta, \tau)-x_{\xi}(t, \xi, \eta, \tau+h) \\
& W(t)=y_{\xi}(t, \xi, \eta, \tau)-y_{\xi}(t, \xi, \eta, \tau+h)
\end{aligned}
$$

are the solutions of a system (10), where by (9) and (15) 


$$
\|x\|_{2},\|\zeta\|_{2} \leq 4 L N\left(1+2 C K e^{N h}\right) h .
$$

Since the coefficients of (14) are bounded by. $N$ the scalar function

$$
\lambda(t)=\left|\tilde{X}_{1}(t)\right|+2\left|\tilde{y}_{1}(t)\right|
$$

satisfies the integral inequality

$$
\lambda(t) \leq \lambda(\tau+h)+3 N \int_{t}^{\tau+h} \lambda(s) d s .
$$

Moreover $\lambda(\tau+h) \leq 2$ by (15), and so by Gronwall's inequality

$$
\lambda(t) \leq 2 e^{3 N(\tau+h-t)} \text { for } \tau \leq t \leq \tau+h .
$$

Since

$$
z(\tau)=I-\tilde{X}_{1}(\tau)=\int_{\tau}^{\tau+h}\left(\tilde{F}_{1} \tilde{X}_{1}+\tilde{F}_{2} \tilde{Y}_{1}\right) d t
$$

it follows that

$$
|Z(\tau)| \leq N \int_{\tau}^{\tau+h} 2 e^{3 N(\tau+h-t)} d t \leq 2 N e^{3 N h} h
$$

Similarly we obtain

$$
\left|\tilde{Y}_{1}(\tau+h)-\tilde{Y}_{1}(\tau)\right| \leq 2 N e^{3 N h} h
$$

We have

$$
\begin{aligned}
P(\tau) W(\tau) & =-P(\tau) \tilde{Y}_{1}(\tau) \\
& =P(\tau)\left[\tilde{Y}_{1}(\tau+h)-\tilde{Y}_{1}(\tau)\right]-[P(\tau)-P(\tau+h)] \tilde{Y}_{1}(\tau+h)
\end{aligned}
$$

and

$$
P(\tau)-P(\tau+h)=\left[I-Y(\tau+h) Y^{-1}(\tau)\right] P(\tau)-P(\tau+h)\left[I-Y(\tau+h) Y^{-1}(\tau)\right] .
$$

Since $|P(\tau)| \leq K$ and

$$
\begin{aligned}
\left|Y(\tau+h) Y^{-1}(\tau)-I\right| & =\left|\int_{\tau}^{\tau+h} A(t) Y(t) Y^{-1}(\tau) d t\right| \\
& \leq \int_{\tau}^{\tau+h} N e^{N(t-\tau)} d t \\
& \leq N e^{N h},
\end{aligned}
$$


it follows that

$$
|P(\tau) W(\tau)| \leq 2 N K e^{3 N h_{h}}+2 N K e^{N h} h\left|\tilde{Y}_{1}(\tau+h)\right| .
$$

But by (15), $\quad\left|\tilde{Y}_{1}(\tau+h)\right| \leq 2 C^{-1} \leq \frac{1}{2}$. Hence

$$
|P(\tau) W(\tau)| \leq 3 N K e^{3 N h} h .
$$

It now follows from (13) that for $0 \leq h \leq h_{0}$ ( $\leq 1$ ).

$$
\|z\|_{2}+c_{2}\|w\|_{2}=O(h)
$$

where the constant involved in the 0-notation depends only on $N, K, L, \alpha, \beta$. Thus, for $0 \leq h \leq h_{0}$,

$$
\begin{aligned}
& \left|x_{\xi}(t, \xi, n, \tau+h)-x_{\xi}(t, \xi, n, \tau)\right|=o(h) e^{2 \beta(t-\tau)}, \\
& \left|y_{\xi}(t, \xi, n, \tau+h)-y_{\xi}(t, \xi, n, \tau)\right|=0(h) e^{2 \beta(t-\tau)} .
\end{aligned}
$$

Similarly we obtain

$$
\begin{aligned}
& \left|x_{n}(t, \xi, n, \tau+h)-x_{n}(t, \xi, n, \tau)\right|=O(h) e^{2 \beta(t-\tau)}, \\
& \left|y_{n}(t, \xi, n, \tau+h)-y_{\eta}(t, \xi, n, \tau)\right|=O(h) e^{2 \beta(t-\tau)} .
\end{aligned}
$$

Now let us look at the function

$$
\psi(\tau, \xi, n)=y(\tau, \xi, n, \tau) .
$$

We have

$$
|\psi(\tau, \xi, n)| \leq \mu,
$$

the partial derivatives $\psi_{\xi}, \psi_{\eta}$ exist and

$$
\begin{gathered}
\left|\psi_{\xi}(\tau, \xi, n)\right| \leq 2 C^{-1}, \quad\left|\psi_{n}(\tau, \xi, \eta)\right| \leq 2 K, \\
\left|\psi_{\xi}\left(\tau, \xi_{1}, n_{1}\right)-\psi_{\xi}\left(\tau, \xi_{2}, \eta_{2}\right)\right| \leq D\left[\left|\xi_{1}-\xi_{2}\right|+C K\left|n_{1}-n_{2}\right|\right] \\
\left|\psi_{\eta}\left(\tau, \xi_{1}, n_{1}\right)-\psi_{n}\left(\tau, \xi_{2}, n_{2}\right)\right| \leq C K D\left[\left|\xi_{1}-\xi_{2}\right|+C K\left|n_{1}-n_{2}\right|\right] .
\end{gathered}
$$

We now show that the partial derivatives $x_{\tau}(t, \xi, n, \tau)$ and $y_{\tau}(t, \xi, n, \tau)$ exist. Let $x_{3}(t), y_{3}(t)$ denote the solution in $B$ of the vector system (14), with 


$$
\begin{aligned}
x_{3}(\tau) & =-f[\tau, \xi, \psi(\tau, \xi, \eta)], \\
P(\tau) y_{3}(\tau) & =-P(\tau)\{A(\tau) \eta+g[\tau, \xi, \psi(\tau, \xi, \eta)]\} .
\end{aligned}
$$

By the superposition principle and the initial conditions for the partial derivatives $x_{\xi}, y_{\xi}, x_{\eta}, y_{\eta}$ we have

$$
\begin{aligned}
& x_{3}(t)=-x_{\xi}(t, \xi, n, \tau) f[\tau, \xi, \psi(\tau, \xi, n)] \\
&-x_{n}(t, \xi, \eta, \tau)\{A(\tau) n+g[\tau, \xi, \psi(\tau, \xi, \eta)]\} \\
& y_{3}(t)=-y_{\xi}(t, \xi, \eta, \tau) f[\tau, \xi, \psi(\tau, \xi, \eta)] \\
& \\
& \quad-y_{n}(t, \xi, \eta, \tau)\{A(\tau) \eta+g[\tau, \xi, \psi(\tau, \xi, \eta)]\} .
\end{aligned}
$$

Put

$$
\begin{aligned}
& z(t)=x(t, \xi, \eta, \tau+h)-x(t, \xi, \eta, \tau) \\
& w(t)=y(t, \xi, \eta, \tau+h)-y(t, \xi, \eta, \tau),
\end{aligned}
$$

and set

$$
\varphi(t)=z(t)-h x_{3}(t), \psi(t)=w(t)-h y_{3}(t) .
$$

Then

$$
\begin{aligned}
& \varphi^{\prime}(t)=F_{1}(t)_{\varphi}(t)+F_{2}(t) \psi(t)+\chi(t), \\
& \psi^{\prime}(t)=G_{1}(t)_{\varphi}(t)+\left[A(t)+G_{2}(t)\right] \psi(t)+\zeta(t),
\end{aligned}
$$

where

$$
|x(t)|,|\zeta(t)| \leq L[|z(t)|+|w(t)|]^{2} .
$$

Therefore, by (9),

$$
\|x\|_{2},\|\zeta\|_{2}=o\left(h^{2}\right) \text { for } h \rightarrow 0
$$

We have

$$
\varphi(\tau)=x(\tau, \xi, \eta, \tau+h)-\xi+f[\tau, \xi, \psi(\tau, \xi, \eta)] h
$$

$P(\tau) \psi(\tau)=P(\tau) y(\tau, \xi, \eta, \tau+h)-P(\tau) \eta+P(\tau)\{A(\tau) n+g[\tau, \xi, \psi(\tau, \xi, \eta)]\} h$. Now, by (9), 


$$
\begin{aligned}
x(\tau, \xi, n, \tau+h)-\xi & =-\int_{\tau}^{\tau+h} f[s, x(s, \xi, \eta, \tau+h), y(s, \xi, n, \tau+h)] d s \\
& =-\int_{\tau}^{\tau+h} f[s, x(s, \xi, \eta, \tau), y(s, \xi, \eta, \tau)] d s+o\left(h^{2}\right) \\
& =-\int_{\tau}^{\tau+h} f[s, \xi, \psi(\tau, \xi, n)] d s+o\left(h^{2}\right) \\
& =-f[\tau, \xi, \psi(\tau, \xi, \eta)] h+o(h) .
\end{aligned}
$$

Also

$$
\begin{aligned}
y(t, \xi, \eta, \tau+h) & =Y(t) P Y^{-1}(\tau+h) \eta \\
+ & \int_{\tau+h}^{t} Y(t) P Y^{-1}(s) g[s, x(s, \xi, \eta, \tau+h), y(s, \xi, n, \tau+h)] d s \\
& -\int_{t}^{\infty} Y(t)(I-P) Y^{-1}(s) g[s, x(s, \xi, \eta, \tau+h), y(s, \xi, \eta, \tau+h)] d s
\end{aligned}
$$

and hence

$$
\begin{aligned}
P(\tau) y(\tau, \xi, n, \tau+h) & =P(\tau) Y(\tau) Y^{-1}(\tau+h) \eta \\
& -\int_{\tau}^{\tau+h} Y(\tau) P Y^{-1}(s) g[s, x(s, \xi, n, \tau+h), y(s, \xi, n, \tau+h)] d s .
\end{aligned}
$$

Since

$$
Y(\tau) Y^{-1}(\tau+h)=I-A(\tau) h+o(h)
$$

it follows that

$P(\tau) y(\tau, \xi, \eta, \tau+h)=P(\tau)[I-A(\tau) h] \eta-P(\tau) g[\tau, \xi, \psi(\tau, \xi, \eta)] h+o(h)$. Thus $\varphi(\tau)=o(h), P(\tau) \psi(\tau)=o(h)$. Applying the inequality (13) we obtain

$$
\|\varphi\|_{2}+C_{2}\|\psi\|=o(h) \text {. }
$$

By the definition of $\varphi$ and $\psi$ this shows that the partial derivatives $x_{\tau}(t, \xi, \eta, \tau), y_{\tau}(t, \xi, \eta, \tau)$ exist and equal $x_{3}(t), y_{3}(t)$ respectively.

It follows that $\psi_{\tau}(\tau, \xi, \eta)$ exists and, by $(21)$, is equal to 


$$
\begin{aligned}
& A(\tau) y(\tau, \xi, \eta, \tau)+g[\tau, x(\tau, \xi, \eta, \tau), y(\tau, \xi, \eta, \tau)] \\
&-y_{\xi}(\tau, \xi, \eta, \tau) f[\tau, \xi, \psi(\tau, \xi, n)] \\
&-y_{n}(\tau, \xi, \eta, \tau)\{A(\tau) \eta+g[\tau, \xi, \psi(\tau, \xi, \eta)]\} .
\end{aligned}
$$

Hence $\psi(\tau, \xi, \eta)$ is a solution of the partial differential equation (22) $\psi_{\tau}+\psi_{\xi} f(\tau, \xi, \Psi)+\psi_{\eta}\{A(\tau) n+g(\tau, \xi, \psi)\}=A(\tau) \psi+g(\tau, \xi, \psi)$. We can now conclude that

$$
\begin{aligned}
\left|\psi_{\tau}(\tau, \xi, n)\right| & =o(1), \\
\left|\psi_{\tau}\left(\tau, \xi_{1}, n_{1}\right)-\psi_{\tau}\left(\tau, \xi_{2}, n_{2}\right)\right| & =o\left[\left|\xi_{1}-\xi_{2}\right|+\left|n_{1}-n_{2}\right|\right] .
\end{aligned}
$$

Also

$\psi_{\xi}(\tau+h, \xi, \eta)-\psi_{\xi}(\tau, \xi, \eta)=y_{\xi}(\tau+h, \xi, n, \tau+h)-y_{\xi}(\tau, \xi, \eta, \tau+h)$

$$
+y_{\xi}(\tau, \xi, \eta, \tau+h)-y_{\xi}(\tau, \xi, \eta, \tau) \text {. }
$$

From the differential equation satisfied by $y_{\xi}$ and from (19) and (15) we obtain for $0 \leq h \leq h_{0}$

$$
\begin{aligned}
\mid y_{\xi}(\tau+h, \xi, n, \tau+h) & -y_{\xi}(\tau, \xi, n, \tau+h) \mid \\
& \leq N \int_{\tau}^{\tau+h}\left[\left|x_{\xi}(s, \xi, n, \tau+h)\right|+2\left|y_{\xi}(s, \xi, \eta, \tau+h)\right|\right] d s \\
& =N \int_{\tau}^{\tau+h}\left[\left|x_{\xi}(s, \xi, n, \tau)\right|+2\left|y_{\xi}(s, \xi, n, \tau)\right|\right] d s+o(h) \\
& =O(h) .
\end{aligned}
$$

Therefore, by (19) again,

$$
\left|\psi_{\xi}(\tau+h, \xi, n)-\psi_{\xi}(\tau, \xi, n)\right|=O(h) .
$$

This inequality has been established for $0 \leq h \leq h_{0}$ but then extends, with the same constant, to arbitrary $h>0$. Similarly we have

$$
\left|\psi_{\eta}(\tau+h, \xi, \eta)-\psi_{\eta}(\tau, \xi, \eta)\right|=O(h) .
$$

The four Lipschitz conditions show that $\psi_{\xi}, \psi_{\eta}$ are continuous functions of $(\tau, \xi, \eta)$, and hence $\psi_{\tau}$ is also by the partial differential 
equation (22).

Altogether we have proved

THEOREM 2. Under the hypotheses of Theorem I there exists a bounded continuous function $\psi(t, x, y)$ defined for $t \geq \tau,|x|<\infty$, $|y|<\mu / 4 K$ with bounded continuous partial derivatives such that $\psi_{t}$ satisfies a Lipschitz condition in $(x, y)$ and $\psi_{x}, \psi_{y}$ satisfy Lipschitz conditions in $(t, x, y)$. This function has the property that if $x(t)$, $y(t)$ is a solution of the system

$$
\begin{aligned}
& x^{\prime}=f(t, x, y) \\
& y^{\prime}=A(t) y+g(t, x, y)
\end{aligned}
$$

for which $x(\tau)=\xi,|y(\tau)|<\mu / 4 K$ then $|y(t)| \leq \mu$ for $t \geq \tau$ if and only if $y(\tau)=\psi(\tau, \xi, \eta)$ for some $n$ such that $|n|<\mu / 4 K$.

For each fixed pair $(\tau, \xi)$ the set $M(\tau, \xi)$ of all points

$\psi(\tau, \xi, n)$ with $|\eta|<\mu / 4 K$ is a $C^{1}$-submanifold of $R^{n}$ with dimension equal to the rank of $P$. In fact, since $\psi\left(\tau, \xi, \eta_{1}\right)=\psi\left(\tau, \xi, n_{2}\right)$ if $P(\tau) n_{1}=P(\tau) n_{2}$ we can restrict attention to $n$ such that $P(\tau) n=n$. Then the mapping $\eta \rightarrow \psi(\tau, \xi, n)$ is continuously differentiable and, since $P(\tau) \psi(\tau, \xi, \eta)=\eta$, it has a continuously differentiable inverse. Thus $M(\tau, \xi)$ is the diffeomorphic image of the intersection of the ball $|n|<\mu / 4 K$ with the subspace of $n$ satisfying $P n=n$.

\section{3.}

We suppose now that the system (1) is defined and satisfies our assumptions over the whole real line. Then, as shown in [1], the system (1) has an integral manifold $y=v(t, x)$, where $v$ is a bounded continuous function with bounded continuous partial derivatives. We will first derive more precise estimates for the function $v$ and its partial derivative $v_{x}$ than were given in [1].

The function $y(t)=y(t, \xi, \tau)$ is the unique bounded solution of the equation

$$
y^{\prime}=A(t) y+\varphi(t)+\psi(t)
$$

where 


$$
\begin{aligned}
\varphi(t) & =g[t, x(t, \xi, \tau), 0]+g_{y}[t, x(t, \xi, \tau), 0] y(t, \xi, \tau), \\
\psi(t) & =G[t, x(t, \xi, \tau), y(t, \xi, \tau)] .
\end{aligned}
$$

For any function $f(t)$ write

$$
|f|=\sup _{-\infty<t<\infty}|f(t)| .
$$

Then

$$
|\psi| \leq \frac{1}{2} \mu L|y|
$$

Also, by Lemma 7 of [1] with $\beta=0$,

$$
\left|\int_{t}^{t+h} \varphi(s) d s\right| \leq p_{0}+p_{0}\left(|y|+\left|y^{\prime}\right|\right)
$$

for $|h| \leq 1$ and either $t \geq \tau, h>0$ or $\tau \geq t, h<0$. So, by Lemmas 4 and 5 of [1] with $\beta=0$, and by the superposition principle,

$$
|y| \leq \alpha^{-1} K \cdot \frac{1}{2} \mu L|y|+4 N K \gamma p_{0}\left(1+|y|+\left|y^{\prime}\right|\right) \text {. }
$$

Since $\left|y^{\prime}\right| \leq N(|y|+1)$ it follows that

$$
\begin{aligned}
|y| & \leq \frac{1}{2} \alpha^{-1} \mu L K|y|+8 N^{2} K \gamma p_{0}(1+|y|) \\
& \leq \frac{1}{2}|y|+8 N^{2} K \gamma p_{0} .
\end{aligned}
$$

Therefore

$$
|y(t, \xi, \tau)| \leq 16 \gamma N^{2} K p_{0} \text { for all } t, \xi, \tau \text {. }
$$

Similarly, if we set

$$
z(t)=x\left(t, \xi_{1}, \tau\right)-x\left(t, \xi_{2}, \tau\right), w(t)=y\left(t, \xi_{1}, \tau\right)-y\left(t, \xi_{2}, \tau\right)
$$

then $w(t)$ is the unique bounded solution of an equation

$$
w^{\prime}=A(t) w+\varphi(t)+\psi(t),
$$

where, by (23),

$$
|\psi(t)| \leq 48 \gamma N^{2} K L p_{0}[|z(t)|+|w(t)|]
$$

and 


$$
\left|\int_{t}^{t+h} \varphi(s) d s\right| \leq p_{0} \beta^{-1}\left(e^{\beta}-1\right)\left[\|z\|+\left\|z^{\prime}\right\|+\|w\|+\left\|w^{\prime}\right\|\right] e^{\beta(t-\tau)}
$$

for $|h| \leq 1$ and either $t \geq \tau, h>0$ or $\tau \geq t, h<0$. Hence, by Lemmas 4 and 5 of [1],

$$
\|w\| \leq 6 p_{0} R^{2} L[\|z\|+\|w\|]+p_{0} R\left[\|z\|+\left\|z^{\prime}\right\|+\|w\|+\left\|w^{\prime}\right\|\right] .
$$

But by (15) of [1]

$$
\|z\|+\|w\| \leq 2\left|\xi_{1}-\xi_{2}\right|
$$

Since

$$
\left\|z^{\prime}\right\| \leq N[\|z\|+\|w\|], \quad\left\|w^{\prime}\right\| \leq 2 N[\|z\|+\|w\|]
$$

it follows that

$$
\|w\| \leq 4 p_{0} R(3 R L+2 N)\left|\xi_{1}-\xi_{2}\right|
$$

Hence

$$
\left|y_{\xi}(t, \xi, \tau)\right| \leq 4 p_{0} R(3 R L+2 N) e^{B|t-\tau|} .
$$

From (23) and (24) we obtain in particular

$$
\begin{gathered}
|v(\tau, \xi)| \leq 16 \gamma N^{2} K p_{0}, \\
\left|v_{\xi}(\tau, \xi)\right| \leq 4 R(3 R L+2 N) p_{0} .
\end{gathered}
$$

It will now be proved that the solutions considered in Theorem 2 of the previous section all converge exponentially to the integral manifold $y=v(t, x)$ as $t \rightarrow \infty$. Let $x(t), y(t)$ be a solution of (1) such that $|y(t)| \leq \mu$ for $t \geq \tau$. By the partial differential equation which $v$ satisfies, (29) of [1],

$$
\begin{aligned}
d v[t, x(t)] / d t= & v_{t}[t, x(t)]+v_{x}[t, x(t)] x^{\prime}(t) \\
=A(t) v[t, x(t)]+g\{t, x(t), v[t, x(t)]\} & -v_{x}[t, x(t)] f\{t, x(t), v[t, x(t)]\} \\
\cdot & +v_{x}[t, x(t)] f\{t, x(t), y(t)\} .
\end{aligned}
$$

Therefore, if we put 


$$
z(t)=v[t, x(t)]-y(t)
$$

we will have

$$
\begin{aligned}
z^{\prime}(t)=A(t) z(t) & +g[t, x(t), y(t)+z(t)]-g[t, x(t), y(t)] \\
& -v_{x}[t, x(t)]\{f[t, x(t), y(t)+z(t)]-f[t, x(t), y(t)]\} .
\end{aligned}
$$

Thus $z(t)$ is a solution of the equation

$$
z^{\prime}=A(t) z+h(t, z),
$$

where

$$
\begin{aligned}
h(t, z)=g[t, x(t), y(t)+z]-g[t, x(t), y(t)] \\
-v_{x}[t, x(t)]\{f[t, x(t), y(t)+z]-f[t, x(t), y(t)]\} .
\end{aligned}
$$

The function $h$ is continuous, vanishes when $z$ vanishes, and has a continuous partial derivative $h_{z}$. Moreover, the properties of $v$ established in [1] imply that

$$
\begin{gathered}
\left|h_{z}(t, z)\right| \leq 2 N, \\
\left|h_{z}\left(t, z_{1}\right)-h_{z}\left(t, z_{2}\right)\right| \leq 2 L\left|z_{1}-z_{2}\right|,
\end{gathered}
$$

while by (26) above

$$
\left|\int_{t_{1}}^{t_{2}} h_{z}(t, 0) d t\right|=O(1) p_{0}+o(1) \mu_{0} \text { for }\left|t_{2}-t_{1}\right| \leq 1 .
$$

If we set

$$
h(t, z)=h_{z}(t, 0) z+H(t, z)
$$

then $|H(t, z)| \leq L|z|^{2}$. It follows from the Lemma proved below that with a suitable choice of $\mu_{0}$ and $q_{0}$

$$
|z(t)| \leq 4 K e^{-\alpha(t-s)}|z(s)| \text { for } t \geq s \geq \tau \text {, }
$$

that is,

(27) $|y(t)-v[t, x(t)]| \leq 4 K e^{-\alpha(t-s)}|y(s)-v[s, x(s)]|$ for $t \geq s \geq \tau$.

LEMMA 3. Let $A(t)$ and $B(t)$ be continuous matrix functions with $|A(t)| \leq N,|B(t)| \leq N$ for $t \geq \tau$ and suppose the linear equation (2) 
has a fundomental matrix $Y(t)$ satisfying (3). Suppose also that

$$
\left|\int_{t_{1}}^{t_{2}} B(t) d t\right| \leq r \text { for }\left|t_{2}-t_{1}\right| \leq 1 \text {, }
$$

and let $f(t, y)$ be a continuous vector function such that

$$
|f(t, y)| \leq \delta|y| \text {. }
$$

If the positive constants $r, \delta$ are so small that $r \leq 1$ and

$$
\theta=\alpha^{-1} K\left[\delta+r\left(3 N+\delta+e^{2 \alpha}\right)\right] \leq \frac{1}{2} \text {, }
$$

then any bounded solution $y(t)$ of the nonlinear equation

$$
y^{\prime}=[A(t)+B(t)] y+f(t, y)
$$

satisfies

$$
|y(t)| \leq 4 K e^{-\alpha(t-s)}|y(s)| \text { for } t \geq s .
$$

In fact any bounded solution $y(t)$ of (28) satisfies

$$
\text { (29) } \begin{aligned}
y(t)=Y(t) P Y^{-1}(s) y(s) & +\int_{s}^{t} Y(t) P Y^{-1}(u)\{B(u) y(u)+f[u, y(u)]\} d u \\
& -\int_{t}^{\infty} Y(t)(I-P) Y^{-1}(u)\{B(u) y(u)+f[u, y(u)]\} d u .
\end{aligned}
$$

Put

$$
\mu(t)=\sup _{u \geq t}|y(u)|
$$

By integrating by parts in the accustomed way we obtain

$$
\left|\int_{t}^{\infty} Y(t)(I-P) Y^{-1}(u) B(u) y(u) d u\right| \leq \frac{1}{2} r \alpha^{-1} K(3 N+\delta+1) \mu(t) .
$$

Similarly, if $s+m \leq t<s+m+1$ then

$$
\begin{aligned}
\left|\int_{s}^{t} y(t) P Y^{-1}(u) B(u) y(u) d u\right| \leq r K \sum_{j=0}^{m} e^{-2 \alpha(t-s-j)}|y(s+j)| & \\
& +r K(3 N+\delta) \int_{s}^{t} e^{-2 \alpha(t-u)}|y(u)| d u
\end{aligned}
$$


But if $j \geq 1$ then

$$
e^{2 \alpha(s+j)}|y(s+j)| \leq e^{2 \alpha} \int_{s+j-1}^{s+j} e^{2 \alpha u} \mu(u) d u .
$$

Hence

$$
\begin{aligned}
\left|\int_{s}^{t} y(t) P Y^{-1}(u) B(u) y(u) d u\right| \leq r K e^{-2 \alpha(t-s)} & |y(s)| \\
& +r K\left(3 N+\delta+e^{2 \alpha}\right) \int_{s}^{t} e^{-2 \alpha(t-u)} \mu(u) d u .
\end{aligned}
$$

Estimating the other terms in (29) in the crudest way we obtain

(30) $|y(t)| \leq(1+r) K e^{-2 \alpha(t-s)}|y(s)|+\theta \alpha \int_{s}^{t} e^{-2 \alpha(t-u)} \mu(u) d u+\frac{1}{2} \theta \mu(t)$.

Choose $h>0$ so large that $8 K e^{-2 \alpha h} \leq 1$. Then, since $\mu$ is a non-increasing function, for $t \geq s+h$

$$
\begin{aligned}
|y(t)| & \leq \frac{1}{4} \mu(s)+\frac{1}{2} \theta \mu(s)+\frac{1}{2} \theta \mu(s) \\
& \leq \frac{3}{4} \mu(s) .
\end{aligned}
$$

Hence $\mu(s+h) \leq \frac{3}{4} \mu(s)$, which shows that $|y(t)| \rightarrow 0$ as $t \rightarrow \infty$.

Therefore for any $t$ there exists $t^{\prime} \geq t$ such that

$$
\mu(t)=\mu(s)=\left|y\left(t^{\prime}\right)\right| \text { for } t \leq s \leq t^{\prime} .
$$

By $(30)$, with $t^{\prime}$ in place of $t$,

$$
\mu(t) \leq 2 K e^{-2 \alpha\left(t^{\prime}-s\right)}|y(s)|+\theta \alpha \int_{s}^{t} e^{-2 \alpha\left(t^{\prime}-u\right)} \mu(u) d u+\theta \mu(t) .
$$

Thus $\varphi(t)=e^{2 \alpha t} \mu(t)$ satisfies

$$
\varphi(t) \leq 4 K e^{2 \alpha s}|y(s)|+\alpha \int_{s}^{t} \varphi(u) d u .
$$

Therefore by Gronwall's inequality,

$$
\varphi(t) \leq 4 K e^{2 \alpha s}|y(s)| e^{\alpha(t-s)} .
$$




\section{Hence}

$$
|y(t)| \leq 4 K e^{-\alpha(t-s)}|y(s)|
$$

Finally it will be shown that the solutions of Theorem 2 are asymptotic not only to the integral manifold but also to particular solutions on the manifold. As in [1] let

$$
k(t, x)=f[t, x, v(t, x)] .
$$

The function $k$ is continuous and has a continuous partial derivative $k_{x}$ such that $\left|k_{x}\right| \leq 2 N$. Let $x(t), y(t)$ be a solution of (I) such that $|y(t)| \leq \mu$ for $t \geq \tau$, and put

$$
\lambda(t)=|y(t)-v[t, x(t)]| .
$$

By (27) we have

$$
\lambda(t) \leq 4 K e^{-\alpha(t-\tau)} \lambda(\tau) \text { for } t \geq \tau \text {. }
$$

Let $x_{n}(t)$ be the solution of the equation

$$
x^{\prime}=k(t, x)
$$

such that $x_{n}(\tau+n)=x(\tau+n)$. Since

$$
x^{\prime}(t)=k[t, x(t)]+\ell(t)
$$

where

$$
\ell(t)=f[t, x(t), y(t)]-f[t, x(t), v[t, x(t)]],
$$

the difference $z_{n}(t)=x(t)-x_{n-1}(t)$ has the representation

$$
z_{n}(t)=\int_{\tau+n-1}^{t}\left\{k[s, x(s)]-k\left[s, x_{n-1}(s)\right]+\ell(s)\right\} d s .
$$

Therefore, since $|\ell(t)| \leq N \lambda(t)$,

$$
\left|z_{n}(t)\right| \leq 2 N \int_{\tau+n-1}^{t}\left|z_{n}(s)\right| d s+N \int_{\tau+n-1}^{t} \lambda(s) d s .
$$

Thus for $\tau+n-1 \leq t \leq \tau+n$ 


$$
\left|z_{n}(t)\right| \leq 2 N \int_{\tau+n-1}^{t}\left|z_{n}(s)\right| d s+\gamma^{-1} R \lambda(\tau) e^{-\alpha n}
$$

and so, by Gronwall's inequality,

$$
\left|x(t)-x_{n-1}(t)\right|=\left|z_{n}(t)\right| \leq \gamma^{-1} R e^{2 N} \lambda(\tau) e^{-\alpha n} .
$$

In particular, for $t=\tau+n$,

$$
\left|x_{n}(\tau+n)-x_{n-1}(\tau+n)\right| \leq \gamma^{-1} R e^{2 N} \lambda(\tau) e^{-\alpha n} .
$$

Therefore, by (15) of [1], for $\tau \leq t \leq \tau+n$

$$
\left|x_{n}(t)-x_{n-1}(t)\right| \leq 2 \gamma^{-1} R e^{2 N} \lambda(\tau) e^{-(\alpha-\beta) n} .
$$

This shows that the sequence $\left\{x_{n}(t)\right\}$ converges uniformly on any bounded subinterval of $[\tau, \infty)$. Let $x_{\infty}(t)$ denote its limit. Suming (32) for $n \geq m$ we get for $\tau \leq t \leq \tau+m$

$$
\begin{aligned}
\left|x_{\infty}(t)-x_{m-1}(t)\right| & \leq 2 \gamma^{-1} R e^{2 N} \lambda(\tau)\left(1-e^{-\alpha / 2}\right)^{-1} e^{-(\alpha-\beta) m} \\
& \leq 4 R e^{2 N} \lambda(\tau) e^{-(\alpha-\beta) m} .
\end{aligned}
$$

Taking $n=m$ in (3I) we deduce that for $\tau+m-1 \leq t \leq \tau+m$

$$
\left|x(t)-x_{\infty}(t)\right| \leq 5 R e^{2 N} \lambda(\tau) e^{-(\alpha-\beta) m} .
$$

Thus for all $t \geq \tau$

$$
\left|x(t)-x_{\infty}(t)\right| \leq 5 R e^{2 N} \lambda(\tau) e^{-(\alpha-\beta)(t-\tau)}
$$

If we set $y_{\infty}(t)=v\left[t, x_{\infty}(t)\right]$ then

$$
\begin{aligned}
\left|y(t)-y_{\infty}(t)\right| & \leq|y(t)-v[t, x(t)]|+\left|v[t, x(t)]-v\left[t, x_{\infty}(t)\right]\right| \\
& =O(1) \lambda(\tau) e^{-(\alpha-\beta)(t-\tau)},
\end{aligned}
$$

by (27) and (33). Altogether we have proved

THEOREM 3. Let the hypotheses of Theorems 1 and 2 hold for $-\infty<t<\infty$ and let $y=v(t, x)$ be the integral manifold whose existence was established in [1]. If $x(t), y(t)$ is a solution of the system (1) such that $|y(t)| \leq \mu$ for $t \geq \tau$ then 


$$
|y(t)-v[t, x(t)]| \leq 4 K e^{-\alpha(t-s)}|y(s)-v[s, x(s)]| \text { for } t \geq s \geq \tau \text {. }
$$

Moreover there exists a solution $x_{\infty}(t), y_{\infty}(t)=v\left[t, x_{\infty}(t)\right]$ of (1) such that for $t \geq \tau$

$$
\left|x(t)-x_{\infty}(t)\right|+\left|y(t)-y_{\infty}(t)\right|=o(1)|y(\tau)-v[\tau, x(\tau)]| e^{-(\alpha-\beta)(t-\tau)} .
$$

4.

My first work on this subject was contained in a joint paper with W.A. Coppel, communicated to the Joumal of Mathematical Analysis and Applications in September 1968. In April 1969 the Editor of the Journal wrote that they had no record of having received the article. Since the earlier treatment has now been superseded by [1], it has not been resubmitted for publication. However, its introduction contained some motivation for the study of the problem and comparison with related work which it seems worthwhile to include here, in a slightly extended form.

The method of averaging, for non-conservative systems, was first applied to some special second order equations by Krylov and Bogolyubov in 1934. Their results were considerably generalized by Bogolyubov in 1945 and given a finished form in Bogolyubov and Mitropolskii [2]. An account of their methods, with numerous applications, was given by Hale [3] and [4]. There are three main results. The first says that solutions of the original equation and solutions of the averaged equation with the same initial point remain close over a large, but finite, interval of time. The second says that if the averaged equation has a constant solution then the original equation has in its neighbourhood a unique bounded solution, with the same stability properties. The third, and most remarkable, says that if the averaged equation has a periodic solution then the original equation has in its neighbourhood a unique integral manifold, with the same stability properties. The introduction of moving orthonormal coordinates near the given periodic orbit reduces the problem to the study of a system (1). The proofs of these results were rather indirect and depended on changes of variables combined with smoothing operations.

It was shown by Gihman [5] in 1952, and by others after him, that the first result is a simple consequence of a theorem about the continuous 
dependence of solutions on a parameter when the variation is not small in the usual sense but is 'integrally small'. A version of his result will be presented here, using Lemma 3 of [1].

THEOREM 4. Let $f(t, x)$ and $g(t, x)$ be continuous functions such that

$$
\begin{aligned}
& \left|f\left(t, x_{1}\right)-f\left(t, x_{2}\right)\right|+\left|g\left(t, x_{1}\right)-g\left(t, x_{2}\right)\right| \leq L\left|x_{1}-x_{2}\right|, \\
& |g(t, x)| \leq N, \\
& \left|\int_{t_{1}}^{t_{2}}\{f(t, x)-g(t, x)\} d t\right| \leq q \text { for }\left|t_{2}-t_{1}\right| \leq 1 .
\end{aligned}
$$

If $x(t)$ and $y(t)$ are solutions of the differential equations

$$
x^{\prime}=f(t, x), y^{\prime}=g(t, y)
$$

for $0 \leq t \leq T$, with $x(0)=y(0)$, then

$$
|x(t)-y(t)| \leq p e^{L t}+p\left(e^{L t}-1\right) / L \text { for } 0 \leq t \leq T \text {, }
$$

provided $q \leq \frac{1}{2} p^{2}(p+L N)^{-1}$.

$$
\begin{aligned}
& \text { If we set } h(t, x)=f(t, x)-g(t, x) \text { then, by Lemma } 3 \text { of [1], } \\
& \qquad\left|\int_{t_{1}}^{t_{2}} h[s, y(s)] d s\right| \leq p \text { for }\left|t_{2}-t_{1}\right| \leq 1 .
\end{aligned}
$$

Therefore

$$
\left|\int_{0}^{t} h[s, y(s)] d s\right| \leq p(t+1) .
$$

The difference $z(t)=x(t)-y(t)$ has the representation

$$
z(t)=\int_{0}^{t}\{f[s, x(s)]-f[s, y(s)]\} d s+\int_{0}^{t} h[s, y(s)] d s .
$$

Hence

$$
|z(t)| \leq L \int_{0}^{t}|z(s)| d s+p(t+1)
$$

and the result follows by the extended Gronwall lemma. 
It was shown by Coppel [6] that the second result of the method of averaging could also be treated, more generally and more directly, as an integrally small perturbation problem. His use of the roughness of exponential dichotomies can be avoided, as in the present paper, and the result thus extended to arbitrary Banach spaces.

The third result, on integral manifolds, has been treated by different methods by a number of authors, e.g. Levinson [7], Diliberto and Hufford [8], Diliberto [9] and [10], Sacker [11] and [12], and Kurzweil [13], [14], [15]. In many cases, however, the problems treated are less general or stronger assumptions are imposed than in the work of Bogolyubov and Mitropolskii. Only Kurzweil has treated the problem as one of integrally small perturbations. The generality of his approach has perhaps obscured some of his contributions. In particular he showed that a solution asymptotic to the integral manifold was also asymptotic to a particular solution on the manifold, which Bogolyubov and Mitropolski proved only in a special case. Our proof of this property was modelled on his. Otherwise this work has been essentially independent of that of Kurzweil. A detailed comparison of the differences in hypotheses, conclusions and methods will not be attempted here.

Sacker's main contribution is connected with higher order smoothness of the manifold. This can also be treated by the present methods. It can be shown that if $f$ and $g$ have $r$ continuous partial derivatives with respect to $x$ and $y$, the $r$-th derivatives being Lipschitzian in $x$ and $y$, then for $q$ sufficiently small, $\partial^{s} v / \partial x^{s}$ and $\partial^{s} v / \partial t \partial x^{s-1}$ exist for $1 \leq s \leq r, \partial^{r} v / \partial x^{r}$ is Lipschitzian in $t$ and $x$ and $\partial^{r} v / \partial t \partial x^{r-1}$ is Lipschitzian in $x$ and continuous in $t$. Also, for $1 \leq s \leq r$, $\left|\partial^{s} v / \partial x^{s}\right|=O(1) q^{2-s}$.

In order to obtain the latter estimates we need the integral smallness of the higher derivatives. However, it is not necessary to impose this as an additional hypothesis since it follows from the assumptions already made. For let us suppose that $h(t, x)$ has a continuous partial derivative $h_{x}(t, x)$ such that $\left|h_{x}\left(t, x_{1}\right)-h_{x}\left(t, x_{2}\right)\right| \leq L\left|x_{1}-x_{2}\right|$ and 


$$
\left|\int_{t_{1}}^{t_{2}} h(t, x) d t\right| \leq q \text { if }\left|t_{1}-t_{2}\right| \leq 1 .
$$

Then if

$$
R=h(t, x+k)-h(t, x)-h_{x}(t, x) k,
$$

$|R| \leq L|k|^{2}$. If we assume $q \leq q_{1}^{2} / 8 L$ and take $k$ to be any vector with $|k|=q_{1} / 2 L$ then for $\left|t_{2}-t_{1}\right| \leq 1$

$$
\begin{aligned}
\left|\int_{t_{1}}^{t_{2}} h_{x}(t, x) d t k\right| & \leq 2 q+L|k|^{2} \\
& \leq q_{1}^{2} / 4 L+q_{1}^{2} / 4 L=q_{1}^{2} / 2 L .
\end{aligned}
$$

Hence

$$
\left|\int_{t_{1}}^{t_{2}} h_{x}(t, x) d t\right| \leq q_{1} \text { for }\left|t_{2}-t_{1}\right| \leq 1 .
$$

This was suggested by Kurzweil [13], Lemma 1.1. It also shows that in [1] the assumption that $g_{x}$ is integrally small is redundant.

Finally as a by-product, the present methods yield a generalization of Bogolyubov's and Mitropolski i's theorem. They assumed that the averaged equation

$$
x^{\prime}=\varepsilon X_{0}(x)
$$

has a solution $\xi(\varepsilon t)$ with period $2 \pi / \varepsilon$ such that the variational equation

$$
x^{\prime}=X_{0}^{\prime}[\xi(t)] x
$$

has $(n-1)$ characteristic exponents with nonzero real parts. This can be replaced by the assumption that the averaged equation has a bounded solution $\xi(\varepsilon t)$ such that the variational equation has a fundamental matrix $Z(t)$ satisfying

$$
\begin{array}{ll}
\left|Z(t) P_{1} z^{-1}(s)\right| \leq M e^{-\sigma(t-s)} & \text { if } s \leq t, \\
\left|Z(t) P_{2} Z^{-1}(s)\right| \leq M e^{-\sigma(s-t)} & \text { if } s \geq t,
\end{array}
$$

where $M, \sigma$ are positive constants and $P_{1}, P_{2}$ are mutually orthogonal 
projections such that $P_{1}+P_{2}$ has rank $n-1$.

Under these assumptions the existence of the integral manifold can be established and almost periodic properties of $\xi(t)$ induce similar properties in the manifold.

\section{References}

[1] W.A. Coppel and K.J. Palmer, "Averaging and integral manifolds", BuZZ. Austraz. Math. Soc. 2 (1970), 197-222.

[2] N.N. Bogolyubov and Yu.A. Mitropolski i, Asymptotic methods in the theory of nonlinear oscizlations (Russian), 3rd ed. (Gos. Izdat. Fiz.-Mat. Lit., Moscow, 1963). English transl. 2nd ed. (Gordon \& Breach, New York, 1962).

[3] Jack K. Hale, "Integral manifolds of perturbed differential systems", Ann. of Math. (2) 73 (1961), 496-531.

[4] Jack K. Hale, Oscizzations in nonZinear systems (McGraw-Hill, New York, Toronto, London, 1963).

[5] I.l. Gihman, "Concerning a theorem of N.N. Bogolyubov", (Russian), Ukrain. Mat. ̌̈. 4 (1952), 215-219.

[6] W.A. Coppel, "Dichotomies and reducibility (II)", J. Differential Equations 4 (1968), 386-398.

[7] Norman Levinson, "Small periodic perturbations of an autonomous system with a stable orbit", Ann. of Math. (2) 52 (1950), $729-738$.

[8] S.P. Diliberto and G. Hufford, "Perturbation theorems for non-linear ordinary differential equations", Contributions to the theory of nonlinear oscillations, 3, 207-236. (Annals of Mathematics Studies, 36, Princeton University Press, Princeton, New Jersey, 1956).

[9] Stephen P. Diliberto, "Perturbation theorems for periodic surfaces I. Definitions and main theorems", Rend. Circ. Mat. Palermo (2) $9(1960), 265-299$. 
[10] Stephen P. Diliberto, "Perturbation theorems for periodic surfaces. II", Rend. Cir. Mat. Palermo (2) 10 (1961), 111-161.

[11] Robert J. Sacker, "A new approach to the perturbation theory of invariant surfaces", Comm. Pure Appl. Math. 18 (1965), 717-732.

[12] Robert J. Sacker, "A perturbation theorem for invariant manifolds and Hölder continuity", J. Math. Mech. 18 (1969), 705-762.

[13] Jaroslav Kurzweil, "Exponentially stable integral manifolds, averaging principle and continuous dependence on a parameter", Czechoslovak Math. J. 16 (91) (1966), 380-423.

[14] Jaroslav Kurzweil, "Invariant manifolds for flows", Differential Equations and Dynamical Systems (Proc. Internat. Sympos., Mayaguez, P.R., 1965, 431-468. Academic Press, New York, 1967).

[15] Jaroslav Kurzweil, "Invariant manifolds for differential systems", (Russian), Differencial'nye Uravnenija 4 (1968), 785-797.

Australian National University,

Canberra, ACT. 